\title{
Evaluation of efficacy of Valsalva maneuver for attenuating propofol injection pain: a prospective, randomized, single blind, placebo controlled study
}

\author{
Sanjay Kumar ${ }^{1}$, Sandeep Khuba ${ }^{1}$, Anil Agarwal ${ }^{1}$, Sujeet Gautam ${ }^{1}$, \\ Madhulika Yadav ${ }^{2}$, and Aanchal Dixit ${ }^{1}$ \\ ${ }^{1}$ Department of Anesthesiology, Sanjay Gandhi Postgraduate Institute of Medical Sciences, Lucknow, ${ }^{2}$ Department \\ of Dentistry, Government Medical College, Kannauj, India
}

Background: Pain on injection is a limitation with propofol use. The effect of the Valsalva maneuver on pain during propofol injection has not been studied. This maneuver reduces pain through the sinoaortic baroreceptor reflex arc and by distraction. We aimed to assess the efficacy of the Valsalva maneuver in reducing pain during propofol injection.

Methods: Eighty American Society of Anesthesiologists class I adult patients undergoing general anesthesia were enrolled and divided into two groups of 40 each. Group I (Valsalva) patients blew into a sphygmomanometer tube raising the mercury column up to $30 \mathrm{mmHg}$ for 20 seconds, while Group II (Control) patients did not. Anesthesia was induced with $1 \%$ propofol immediately afterwards. Pain was assessed on a 10-point visual analog scale (VAS), where 0 represented no pain, and 10, the worst imaginable pain, and a 4-point withdrawal response score, where 0 represented no pain, and 3 , the worst imaginable pain. Scores were presented as median (interquartile range).

Results: We analyzed the data of 70 patients. The incidence of pain was significantly lower in the Valsalva than in the control group ( $53 \%$ vs. $78 \%, \mathrm{P}=0.029$ ). The withdrawal response score was significantly lower in the Valsalva group (1.00 $[0.00-1.00]$ vs. 2.00 [2.00-3.00], $\mathrm{P}<0.001)$. The VAS score was significantly lower in the Valsalva group $(1.00$ [0.00-4.00] vs. 7.00 [6.25-8.00], $\mathrm{P}<0.001)$.

Conclusions: A prior Valsalva maneuver is effective in attenuating injection pain due to propofol; it is advantageous in being a non-pharmacological, safe, easy, and time-effective technique.

Keywords: Distraction; General anesthesia; Propofol; Valsalva maneuver; Visual analog scale; Withdrawal response score.

Corresponding author: Sanjay Kumar, M.D.

Department of Anesthesiology, Sanjay Gandhi Postgraduate Institute of Medical Sciences, Raebareli Road, Lucknow 226014, India

Tel: 91-9452535221, Fax: 91-522-2668017

Email: drsanjaygupta9@gmail.com

ORCID: https://orcid.org/0000-0003-2875-5838

Received: January 18, 2018.

Revised: March 31, 2018 (1st); March 15, 2018 (2nd); May 15, 2018

(3rd); May 18, 2018 (4th); May 23, 2018 (5th).

Accepted: May 23, 2018.

Korean J Anesthesiol 2018 December 71(6): 453-458

https://doi.org/10.4097/kja.d.18.00017

\section{Introduction}

Propofol is a commonly used anesthetic for inducing general anesthesia [1]. Pain on propofol injection is distressing and is one of the limitations of its use. Propofol is a phenol compound, which irritates nerve endings on the venous endothelium to produce immediate pain, whereas delayed pain is mediated by release of bradykinin [2]. Bradykinin causes venodilation and increases venous permeability, thereby facilitating contact of the aqueous phase of propofol with nerve endings. Delayed pain

(c) This is an open-access article distributed under the terms of the Creative Commons Attribution Non-Commercial License (http://creativecommons.org/ licenses/by-nc/4.0/), which permits unrestricted non-commercial use, distribution, and reproduction in any medium, provided the original work is properly cited. 
occurs 10-20 seconds after injection [2]. Pain due to propofol injection causes somatic disturbances in patients.

Various methods have been tried so far to reduce pain on propofol injection. A commonly used technique is with lignocaine either as pre-treatment or mixed with propofol [2]. Other methods include the use of butorphanol, ondansetron, metoclopramide, opioid, or thiopentone. Propofol injection into a large vein, pre-injection cooling, or warming of propofol have also been investigated [3-6]. However, these methods have been unable to address the psychological impact of pain during propofol injection. The Valsalva maneuver is a physiological technique, used in the reduction of pain associated with several procedures [7-9]. The Valsalva maneuver alleviates both the somatic and psychological aspects of painful procedures $[7,8]$. This maneuver reduces pain through the sinoaortic baroreceptor reflex arc and by distraction. Pain induced psychological disturbances include anxiety, depression, dissatisfaction, and post-traumatic stress disorder (PTSD). Poorly managed needle-prick pain may lead to decreased pain threshold for future needle procedures [10]. No previous study has cited the use of the Valsalva maneuver for the reduction of pain on propofol injection. Therefore, we planned a study to evaluate the analgesic efficacy of the Valsalva maneuver in alleviating pain during propofol injection.

\section{Materials and Methods}

This randomized, single-blind, placebo-controlled prospective study was conducted in urology operating room complex, from October 2015 to March 2016. Prior institutional ethics committee approval (Ref: 2015/101/IP/86) and written informed consent were obtained from patients. The study is registered with the Clinical Trials Registry of India (Ref: CTRI: 2016:12:007618).

\section{Inclusion criteria}

We enrolled 80 adult patients aged 18-60 years, of American Society of Anesthesiologists (ASA) grade I physical status. Patients scheduled for elective urological surgery under general anesthesia with propofol as an induction agent, were randomly allocated to two groups of 40 each using a computer-generated table of random numbers.

\section{Exclusion criteria}

Patients with mental illness, restlessness, cardio-respiratory disease, chronic pain disorder, use of preoperative analgesic medication, history of propofol allergy, history of drug abuse, and inability to perform the Valsalva maneuver were excluded from the study.

\section{Sample size estimation}

Based on previous studies, the incidence of pain on propofol injection was assumed to be $70 \%$; we considered a $40 \%$ reduction in pain to be clinically significant [11]. Twenty-nine patients were calculated as the minimum sample size for each group assuming a type I error of 0.05 and a desired power value of $90 \%$ using the two-tailed $\mathrm{Z}$ test for proportions. The significance level of the test was targeted at 0.05 . To detect the above difference in proportions, the calculated effect size was 0.823 . The sample size was calculated using power analysis and the PASS sample size software (version 8; NCSS Statistical Software, USA). Considering possible dropouts, 40 patients were included in each group.

\section{Study randomization, intervention and blinding}

A computer-generated random allocation sequence, concealed in 80 consecutively numbered, sealed envelopes, mentioning group distribution, was prepared by a ward nurse not involved in the trial. Subjects were administered oral lorazepam $2 \mathrm{mg}$ with sips of water on the night before surgery. Intravenous cannulation with an $18 \mathrm{G}$ cannula was performed on the dorsum of the non-dominant hand in the preoperative room $1 \mathrm{~h}$ before the scheduled operation time. Patients were scheduled as the first case between 9-11 am to prevent the effect of the circadian difference on pain perception. The assigned envelopes were opened by enrolled patients on the morning of surgery for group allocation. Monitoring was commenced using electrocardiography, blood pressure measurements, pulse oximetry, and capnography. An anesthesia technician, otherwise not involved in the study, loaded 1\% propofol-Long Chain Triglycerides (LCT) (Fresenius Kabi, Austria) into a $50 \mathrm{ml}$ syringe. Patients allocated to perform the Valsalva maneuver (Group I, Valsalva group) blew into a rubber tubing connected to a sphygmomanometer and raised the mercury column to $30 \mathrm{mmHg}$ for a period of at least 20 seconds; the control group (Group II) placed the tube between the lips, but did not perform the maneuver. All patients received one-fourth of the total calculated dose of $1 \%$ propofol-LCT over 5 seconds immediately after the group-specific intervention as the initial dose. Following this, we ceased the infusion for 15 seconds. We started assessment of pain 20 seconds after starting propofol administration. The remaining three-fourths of the dose was administered after 20 seconds over 1 minute, before the patients lost consciousness. The data nurse who assessed pain levels was blinded to the groups in the following manner. A 3-feet high curtain was placed transversely across at the level of the costal margin with the patient lying supine. The technician who administered propofol and the nurse who collected pain data were unaware of the group-specific inter- 
vention as the head of the patient was concealed from them by the curtain. Immediately after the group-specific intervention, the data collection nurse moved to the head end of the patient (the other side of the curtain) for pain assessment. The intensity of pain was evaluated using a ruler as the visual analogue scale (VAS) and a questionnaire for assessment of the withdrawal response score. A stopwatch was used to record the time duration of propofol injection in seconds. The data collection nurse performed hemodynamic monitoring using a monitor placed at a height of 6 feet from the floor and visible from both sides of the curtain. Patients were instructed about the VAS ruler showing numbers on one side and corresponding facial expressions on the opposite side. The VAS score ranged from $0-10$, with 0 indicating no pain and 10 indicating the worst imaginable pain. Patients were instructed to mark a point on the VAS ruler that represented the intensity of pain. The pain score was determined by measuring the distance in mm between 0 (no pain) and the mark indicated by the patient on the ruler.

Twenty seconds after propofol administration, withdrawal response was assessed using standard questions including comfort during injection, verbal response, and behavioral signs (facial grimacing, arm withdrawal, or tears). Pain assessment using the withdrawal response scoring was graded on a 4-point scale: $0=$ no pain, $1=$ mild pain (pain reported only in response to questioning without any behavioral signs), $2=$ moderate pain (pain reported in response to questioning and accompanied by a behavioral signs or pain reported spontaneously without questioning), and 3 = severe pain (strong vocal response or response accompanied by facial grimacing, arm withdrawal, or tears). Hemodynamic parameters including the systolic blood pressure (SBP), the diastolic blood pressure (DBP), and the heart rate (HR) were recorded before the Valsalva maneuver (SBP0, DBP0 and HR0), at 1 minute (SBP1, DBP1 and HR1), at 2 minutes after the maneuver, following propofol injection (SBP2, DBP2 and HR2), and at 5 minutes (SBP5, DBP5 and HR5).

Anesthesia was induced 1 minute after the Valsalva maneuver with intravenous $1 \%$ propofol-LCT $2.5 \mathrm{mg} / \mathrm{kg}$ and fentanyl $3 \mu \mathrm{g} / \mathrm{kg}$. Endotracheal intubation was performed by a dedicated senior resident not involved in the study, after 3-4 minutes of a bolus dose of vecuronium and 5 minutes after propofol administration. General anesthesia was maintained using isoflurane and fentanyl.

VAS and withdrawal response score were assessed by a dedicated anesthesiologist who was blinded to group allocation and was not involved in the study.

On postoperative day 1 , the propofol injection site was checked for signs of inflammation including edema, pain, wheal, and flare by a pain clinic registrar who was not aware of the study and the drug administered.

Online access in http://ekja.org

\section{Statistical analysis}

Continuous data were considered to be normally distributed when the standard deviation was less than half of the mean values. All normally distributed, continuous variables are presented as mean \pm standard deviation (SD) and categorical data as frequency (\%). Ordinal data were presented as median with interquartile range (IQR). To compare the means between or within independent groups, the independent samples $t$-test or the one-way ANOVA test was used. The chi-square test or the Fisher's exact test were used to compare proportions as appropriate. The VAS pain scores and the withdrawal response score were analyzed using the Mann Whitney $U$ test. A P value of < 0.05 was considered significant. The results were analyzed using the unpaired Student's t-test for continuous, and the chi-square or the Fisher's exact test for categorical variables. We used the SPSS 16.0 statistical software package (SPSS Inc., USA) for all analyses.

\section{Results}

Among the eighty patients who were eligible for the study, 76 were included after randomization. Only 70 (87\%) patients completed the study (Fig. 1). Among those excluded, four patients refused to participate in the study as they preferred thiopentone induction. Two patients in Group II were excluded after randomization as they required cannulation of the antecubital vein. Four patients in Group I were excluded from the study following initial randomization and were not considered for further analysis; three patients could not perform the Valsalva maneuver while the other patient required cannulation of the antecubital vein. There was no difference between groups regarding age,

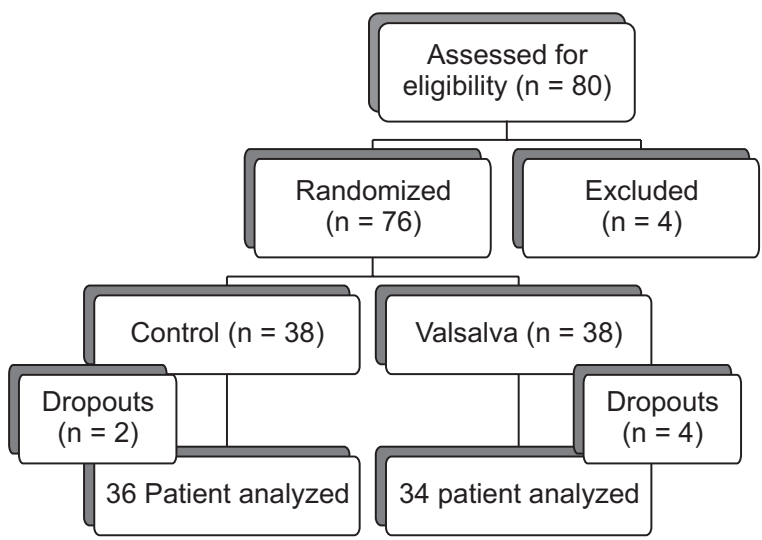

Fig. 1. A flow diagram of the study. Total 80 patients were enrolled for eligibility, out of which 76 patients were posted for study following randomization and 70 patients completed the study. There were 10 dropouts. Total 70 patients were assessed for statistical analysis. 
Table 1. Demographic Data

\begin{tabular}{lccc}
\hline & $\begin{array}{c}\text { Valsalva group } \\
(\mathrm{n}=34)\end{array}$ & $\begin{array}{c}\text { Control group } \\
(\mathrm{n}=36)\end{array}$ & P value \\
\hline Age $(\mathrm{yr})$ & $39.8(12.2)$ & $44.8(13.0)$ & 0.106 \\
Sex $(\mathrm{M} / \mathrm{F})$ & $18 / 16$ & $18 / 18$ & 0.809 \\
Height $(\mathrm{cm})$ & $154.0(8.4)$ & $154.5(8.5)$ & 0.830 \\
Weight $(\mathrm{kg})$ & $62.0(9.5)$ & $59.1(9.5)$ & 0.105 \\
\hline
\end{tabular}

Values are presented as mean (SD) or number of patient.

Table 2. Propofol Pain Incidence and Severity

\begin{tabular}{lccr}
\hline & $\begin{array}{c}\text { Valsalva group } \\
(\mathrm{n}=34)\end{array}$ & $\begin{array}{c}\text { Control group } \\
(\mathrm{n}=36)\end{array}$ & P value \\
\hline Incidence $(\%)$ & $18(53 \%)$ & $28(78 \%)$ & 0.029 \\
Withdrawal score & $1.00[0.00-1.00]$ & $2.00[2.00-3.00]$ & $<0.001$ \\
VAS & $1.00[0.00-4.00]$ & $7.00[6.25-8.00]$ & $<0.001$ \\
\hline
\end{tabular}

Values are presented as percentage or median [interquartile range].

gender distribution, height, and body weight $(\mathrm{P}>0.05)$ (Table 1). Pain incidence and severity were significantly reduced in valsalva group as compared with the control group $(\mathrm{P}<0.05)$ (Table 2$)$. Hemodynamic parameters including systolic and diastolic blood pressure, and heart rates were not significantly different between the two groups $(\mathrm{P}>0.05)$ (Table 3 ).

There was no incidence of fainting, hemodynamic instability, and skin changes in the postoperative period in control and Valsalva groups both.

\section{Discussion}

Our study showed a significant reduction in the incidence as well as the intensity of pain on propofol injection with the use of the Valsalva maneuver.

It is important to avoid pain on propofol injection in adults undergoing anesthesia [12]. Propofol related pain is embarrassing for the anesthetist and distressing for the patient. According to previously published studies, the incidence of pain on propofol injection is approximately $80 \%$ without the use of any prior analgesic [5-7]. The use of remifentanil could reduce the incidence of pain by $4 \%-40 \%$ although it took an additional 60-120 seconds for induction of anesthesia [11]. Even optimal prophylactic techniques, including the use of a tourniquet with lignocaine administration, leads to discomfort during tourniquet application for 30-120 seconds; besides, the maximal effect is only $60 \%$ [6]. Pharmacological methods require both appropriate dosing and timing of drugs before injection for maximum analgesic effect during propofol induction $[6,11]$.

Valsalva maneuver has the advantage of being a simple analgesic technique, with rapid onset and is devoid of pharmacological side effects. Our study did not reveal hemodynamic compli-
Table 3. Hemodynamic Variables

\begin{tabular}{lccc}
\hline & $\begin{array}{c}\text { Valsalva group } \\
(\mathrm{n}=34)\end{array}$ & $\begin{array}{c}\text { Control group } \\
(\mathrm{n}=36)\end{array}$ & P value \\
\hline SBP0 $(\mathrm{mmHg})$ & $125.5(19.3)$ & $128.7(15.8)$ & 0.456 \\
SBP1 $(\mathrm{mmHg})$ & $119.6(12.7)$ & $116.3(15.4)$ & 0.329 \\
SBP2 $(\mathrm{mmHg})$ & $118.4(13.4)$ & $113.6(14.9)$ & 0.163 \\
SBP5 $(\mathrm{mmHg})$ & $121.6(12.4)$ & $115.6(14.3)$ & 0.067 \\
DBP0 $(\mathrm{mmHg})$ & $78.5(6.0)$ & $81.3(6.9)$ & 0.077 \\
DBP1 $(\mathrm{mmHg})$ & $76.8(8.1)$ & $76.2(9.7)$ & 0.800 \\
DBP2 $(\mathrm{mmHg})$ & $78.4(9.8)$ & $78.0(9.0)$ & 0.836 \\
DBP5 $(\mathrm{mmHg})$ & $121.6(12.4)$ & $81.1(8.6)$ & 0.183 \\
HR0 (beats/min) & $80.4(9.8)$ & $83.4(13.1)$ & 0.285 \\
HR1 (beats/min) & $80.4(9.8)$ & $83.4(13.1)$ & 0.285 \\
HR2 (beats/min) & $71.2(8.5)$ & $72.7(9.1)$ & 0.461 \\
HR5 (beats/min) & $80.4(9.8)$ & $83.4(13.1)$ & 0.285 \\
\hline
\end{tabular}

Values are presented as mean (SD). SBP0: systolic blood pressure measured before Valsalva, SBP1: systolic blood pressure measured 1 minute after Valsalva, SBP2, 5: systolic blood pressure measured at 2 and 5 minutes after propofol injection. DBP0: diastolic blood pressure measured before Valsalva, DBP1: diastolic blood pressure measured at 1 minute after Valsalva, DBP2, 5: diastolic blood pressure measured at 2 and 5 minutes after propofol injection. HR0: heart rate at before Valsalva, HR1: heart rate at 1 minute after Valsalva, HR2, 5: heart rate at 2 and 5 minutes after propofol injection.

cations with the use of this maneuver.

Prior studies have used either the VAS or the withdrawal response score for the assessment of pain during propofol injection. VAS is a subjective method of pain assessment while the withdrawal response score is objective. Hence, the withdrawal response score may be a more reliable method of assessment than subjective methods like the VAS, particularly in patients who are sedated with propofol [2]. We found no previously published literature on subjective or objective pain assessment during propofol injection. Hence, we aimed to use both subjective and objective methods of pain assessment using the VAS and the withdrawal response score respectively. VAS is one of the most ideal methods of pain assessment in the clinical scenario [3]; however, VAS seems relatively more sensitive to subtle changes in effect over time with propofol sedation than withdrawal response score. VAS can exaggerate the numerical interpretation of a single subjective report of transient pain during propofol injection [4]. To perform VAS scoring, adequate handeye coordination is required. Hand-eye coordination is the coordinated control of eye movement with hand movement, and the processing of visual input to guide reaching, touching, or grasping, along with the use of hand proprioception to guide the eyes. Due to rapid change in the level of consciousness with propofol administration, hand-eye coordination could be impaired in patients leading to inappropriate interpretation or loss of data. The limitations to the use of the VAS include difficulty in finding the appropriate point on the VAS line, and the inability to complete 
the VAS questionnaire due to the sedative effect of propofol [13]. This may be due to impairment of cognition, or lack of physical capability consequent to altered level of consciousness, which may affect scoring. We chose the withdrawal response score in our study because of its objective nature and the problems encountered during VAS scoring in subjects undergoing propofol induction. Both the VAS score and the withdrawal response score for pain intensity were significantly reduced in the Valsalva group compared to the control group. There were some differences in severity assessment between the two scores; this could be because of the variable nature of the VAS and the withdrawal response scores, and impairment of cognitive and motor ability, due to the sedative effect of propofol. We did not assess the satisfaction level of patients.

Although the Valsalva maneuver appears less efficacious than previously described methods, it is free of side effects and complications. The relative lack of efficacy compared to more optimal methods like lignocaine method could be because of the use of LCT propofol, and the lack of concomitantly administered analgesic drugs. Non-pharmacological methods may also be less efficacious; besides, the duration of the Valsalva maneuver may have been too short for maximum analgesic effect. The Valsalva maneuver is more advantageous because it is simple to perform, physiological, time and cost effective, painless, free of side effects, and patient-friendly. Hence, the Valsalva maneuver may be considered as a good alternative in compliant patients who do not prefer pharmacological intervention. It may be used as an adjuvant to other proven techniques as an analgesic-sparing method to ensure reduction of pain during propofol induction as complete removal of pain cannot be guaranteed; besides, it may help with reduction of dose-related side effects of analgesics.

Previous literature shows that pain has both somatic and psychological components. Somatic components include physical pain and hemodynamic changes. Commonly observed psychological disturbances include anxiety, depression, dissatisfaction, and PTSD. Anxiety and PTSD decrease pain threshold for future procedures [10]. Apart from the use of the antecubital vein for intravenous cannulation, previously established methods on the reduction of pain during propofol injection were pharmacological like lignocaine and opioid use, with a central or peripherally mediated effect. These methods carry drug-specific side-effects and complications related to central and peripheral mechanisms. For example, on some occasions, opioids produce transient chest discomfort, cough, bradycardia, nausea, and itching. The lignocaine-tourniquet method requires tourniquet application for 30-60 seconds for the intervention to exert maximal effect. These pharmacological methods are cumbersome and inhibit only somatic pain; the psychological component remains unaddressed. None of these methods are simple, physiological, and attention diverting by actively involving the subject in the relief of pain.

The Valsalva maneuver is a proven physiological and non-pharmacological method to reduce different types of skin puncture pain including venous cannulation and lumbar puncture for spinal anesthesia [7-9]. The Valsalva maneuver addresses both components of pain unlike previously reported methods of attenuation of propofol pain. In contrast to opioid, ketamine, and local anesthetic pretreatment, the Valsalva maneuver does not produce sedation and hence does not interfere with pain assessment.

The Valsalva maneuver is performed by forced expiration against a closed glottis and holding breath for at least 16 seconds [14]. The somatic mechanism behind the analgesic action of the Valsalva maneuver is due to the rise in intrathoracic pressure; this results in compression of intrathoracic blood vessels that activates the sinoaortic baroreceptor [14]. This sinoaortic baroreceptor reflex arc maintains circulation through peripheral receptors as well as the central nervous system by balancing parasympathetic and sympathetic efferent output to the cardiovascular system. Sinoaortic baroreceptor reflex activation by peripheral cardiovascular stimuli engages the central nervous system simultaneously and inhibits pain conduction. Sinoaortic baroreceptor reflex inhibits pain by release of substance-P and noradrenaline into the circulation. Noradrenaline causes vasoconstriction and increases venous return leading to increased central blood volume $[14,15]$. The distraction component of the Valsalva maneuver attenuates pain by distracting the mind of the subject from the immediate environment or stimulus [14,15]. It manipulates the attention framework and diverts the mind away from pain-producing stimuli in the immediate environment $[15,16]$. Other advantages of distraction include reduction of the level of fear, anxiety, and pain along with increasing the coping ability to procedural pain [17-19]. It reduces pain by activating the segmental pain inhibitory pathways [20]. Previous studies have documented various distraction methods and their role in pain attenuation. The Valsalva maneuver, virtual reality, movies, and balloon inflation have been studied in this context $[7,8,17]$.

Our study has a few limitations. We did not use the lignocaine-tourniquet technique, a proven method for the reduction of pain during propofol injection, as control. There was no double-blinding during the intervention, and we did not assess the satisfaction level of patients. Besides, we did not measure serum triglyceride levels. The analgesic effect of the Valsalva maneuver was not substantiated by measurement of the level of biomarkers.

A future study may be conducted to the compare the analgesic efficacy of the Valsalva maneuver with the proven lignocaine-tourniquet technique.

In conclusion, the Valsalva maneuver may be useful for at- 
tenuating propofol injection pain in ASA class I patients. It is a simple, easy, physiological, non-pharmacological, time and cost effective analgesic method to alleviate the pain of propofol injection into small veins of the hand without producing any significant intra- and postoperative complications.

\section{ORCID}

Sanjay Kumar, https://orcid.org/0000-0003-2875-5838

Sandeep Khuba, https://orcid.org/0000-0002-1840-1131

Anil Agarwal, https://orcid.org/0000-0002-5231-9609

Sujeet Gautam, https://orcid.org/0000-0001-9462-521X

Madhulika Yadav, https://orcid.org/0000-0002-6454-1952

Aanchal Dixit, https://orcid.org/0000-0001-9995-0230

\section{References}

1. Kang JG, Lee SM, Lim SW, Chung IS, Hahm TS, Kim JK, et al. Correlation of AEP, BIS and OAA/S scores under stepwise sedation using propofol TCI in orthopedic patients undergoing total knee replacement arthroplasty under spinal anesthesia. Korean J Anesthesiol 2004; 46: 284-92.

2. Shabana AM. Prevention of propofol injection pain, using lidocaine in a large volume does it make a difference? A prospective randomized controlled double blinded study. Egyptian J Anaesth 2013; 29: 291-4.

3. Scott RP, Saunders DA, Norman J. Propofol: clinical strategies for preventing the pain of injection. Anaesthesia 1988; 43: 492-4.

4. King SY, Davis FM, Wells JE, Murchison DJ, Pryor PJ. Lidocaine for the prevention of pain due to injection of propofol. Anesth Analg 1992; 74: 246-9.

5. McCrirrick A, Hunter S. Pain on injection of propofol: the effect of injectate temperature. Anaesthesia 1990; 45: 443-4.

6. Picard P, Tramèr MR. Prevention of pain on injection with propofol: a quantitative systematic review. Anesth Analg 2000; 90: 963-9.

7. Gupta D, Agarwal A, Dhiraaj S, Tandon M, Kumar M, Singh RS, et al. An evaluation of efficacy of balloon inflation on venous cannulation pain in children: a prospective, randomized, controlled study. Anesth Analg 2006; 102: 1372-5.

8. Agarwal A, Sinha PK, Tandon M, Dhiraaj S, Singh U. Evaluating the efficacy of the valsalva maneuver on venous cannulation pain: a prospective, randomized study. Anesth Analg 2005; 101: 1230-2.

9. Mohammadi SS, Pajand AG, Shoeibi G. Efficacy of the valsalva maneuver on needle projection pain and hemodynamic responses during spinal puncture. Int J Med Sci 2011; 8: 156-60.

10. McMurtry CM, Pillai Riddell R, Taddio A, Racine N, Asmundson GJ, Noel M, et al. Far from "just a poke": common painful needle procedures and the development of needle fear. Clin J Pain 2015; 31(10 Suppl): S3-11.

11. Lee JR, Jung CW, Lee YH. Reduction of pain during induction with target-controlled propofol and remifentanil. Br J Anaesth 2007; 99: 87680.

12. Macario A, Weinger M, Truong P, Lee M. Which clinical anesthesia outcomes are both common and important to avoid? The perspective of a panel of expert anesthesiologists. Anesth Analg 1999; 88: 1085-91.

13. Klimek L, Bergmann KC, Biedermann T, Bousquet J, Hellings P, Jung K, et al. Visual analogue scales (VAS): Measuring instruments for the documentation of symptoms and therapy monitoring in cases of allergic rhinitis in everyday health care: Position Paper of the German Society of Allergology (AeDA) and the German Society of Allergy and Clinical Immunology (DGAKI), ENT Section, in collaboration with the working group on Clinical Immunology, Allergology and Environmental Medicine of the German Society of Otorhinolaryngology, Head and Neck Surgery (DGHNOKHC). Allergo J Int 2017; 26: 16-24.

14. Englstrom JW, Martin JB. Disorders of the autonomic nervous system. In: Principles of Internal Medicine. Edited by Braunwald E, Fauci AS: New York, McGraw-Hill. 2001, pp 2416-21.

15. Randich A, Maixner W. Interactions between cardiovascular and pain regulatory systems. Neurosci Biobehav Rev 1984; 8: 343-67.

16. McCaul KD, Malott JM. Distraction and coping with pain. Psychol Bull 1984; 95: 516-33.

17. Cohen LL. Reducing infant immunization distress through distraction. Health Psychol 2002; 21: 207-11.

18. Cohen LL. Behavioral approaches to anxiety and pain management for pediatric venous access. Pediatrics 2008; 122 Suppl 3: S134-9.

19. Cohen LL, Bernard RS, Greco LA, McClellan CB. A child-focused intervention for coping with procedural pain: are parent and nurse coaches necessary? J Pediatr Psychol 2002; 27: 749-57.

20. Ong EL, Lim NL, Koay CK. Towards a pain-free venepuncture. Anaesthesia 2000; 55: 260-2. 